\title{
Allergic bronchopulmonary aspergillosis and severe asthma with fungal sensitization in patients with uncontrolled asthma: An experience from Southwestern Iran
}

\author{
Mozhgan Moghtaderi ${ }^{1}$, Shirin Farjadian*2, Saeed Hossieni Teshnizi ${ }^{3}$, Maryam Hadibarhaghtalab ${ }^{4}$
}

Received: 23 Aug 2017

Published: 11 Sep 2019

Conflicts of Interest: None declared

Funding: Shiraz University of Medical Sciences

*This work has been published under CC BY-NC-SA 1.0 license.

Copyright $\odot \underline{\text { Iran University of Medical Sciences }}$

Cite this article as: Moghtaderi M, Farjadian S, Hossieni Teshnizi S, Hadibarhaghtalab M. Allergic bronchopulmonary aspergillosis and severe asthma with fungal sensitization in patients with uncontrolled asthma: An experience from Southwestern Iran. Med J Islam Repub Iran. 2019 (11 Sep);33:95. https://doi.org/10.47176/mjiri.33.95

\section{Introduction}

Small fungal spores of the mold Aspergillus fumigatus (AF), which is abundant worldwide, can easily reach the lower airways and alveoli through inhalation $(1,2)$. In patients with asthma, the prevalence of Aspergillus sensitization is reported to be about $2 \%$ and it is more often associated with severe type of asthma (3). The major types of respiratory disease caused by these fungi are Aspergillus-induced asthma (AIA), severe asthma with fungal sensitization (SAFS), and allergic bronchopulmonary aspergillosis (ABPA) in patients with asthma $(4,5)$.

Patients with asthma who have an immediate positive reaction to the Aspergillus allergen without evidence of inflammation or tissue damage are considered to have AIA (2). The term SAFS was introduced in 2006 for patients with asthma who had an immediate positive skin reaction to Aspergillus antigens or elevated serum IgE levels to AF accompanied by frequent exacerbations of respiratory symptoms $(4,5)$.

Corresponding author: Dr Shirin Farjadian, farjadsh@sums.ac.ir

1. Allergy Research Center, Allergy Clinic of Ali-Asghar Hospital, Shiraz University of Medical Sciences, Shiraz, Iran

2. Department of Immunology, Shiraz University of Medical Sciences, Shiraz, Iran

3. Clinical Research Development Center of Children Hospital, Hormozgan University of Medical Sciences, Bandar Abbas, Iran

4. Noncommunicable Disease Research Center, Fasa University of Medical Sciences, Fasa, Iran
ABPA is a complex pulmonary disease characterized by deterioration of lung function, elevated total serum IgE, elevated serum IgE and/or serum IgG to AF, recurrent transient chest X-ray infiltrate $s$, and peripheral eosinophilia; nevertheless, the key sign is skin test reactivity to Aspergillus $(6,7)$. In patients with asthma, ABPA is sometimes diagnosed without evidence of typical proximal bronchiectasis; such cases are considered seropositive ABPA (ABPAS) (6).

Shiraz is a city in Southwestern Iran which is influenced by dust storms originating from Arabic countries. The present study was designed to determine the frequency of sensitization to Aspergillus and the prevalence of ABPA and SAFS in individuals with severe asthma in Southwestern Iran.

This cross sectional study involved adult patients with severe asthma who were referred to the Allergy Clinic Center at Ali-Asghar hospital, affiliated to Shiraz University of

\section{$\uparrow$ What is "already known" in this topic:}

In patients with asthma, the prevalence of Aspergillus sensitization is reported to be $16 \%$ to $45 \%$, severe asthma with fungal sensitization about $33 \%$, and allergic bronchopulmonary aspergillosis from $1 \%$ to $27 \%$ worldwide.

\section{$\rightarrow$ What this article adds:}

In patients with severe asthma in Southwestern Iran, the prevalence of Aspergillus sensitization, severe asthma with fungal sensitization, and allergic bronchopulmonary aspergillosis is $17 \%, 10.2 \%$, and $3.4 \%$, respectively. It seems that airborne fungal spores in outdoor dust are a potential threat to public health in this region, and additional studies of avoidance strategies to prevent fungal sensitization in patients at risk for asthma are a potentially effective means of controlling severe asthma and its complications. 
Medical Sciences in Shiraz, Iran, during a 6-month period from October 2014 to March 2015. All patients were diagnosed with severe asthma according to Expert Panel Report 3 criteria (8). After approval of the study protocol by the Ethics Committee of the university (EC-P-92-6337), written informed consent was obtained from each participant. Patients with a history of tuberculosis, smoking, diabetes mellitus, or hypertension and pregnant women were excluded from the study. Patients with asthma attack who received treatment with an oral glucocorticoid within the previous 6 months and patients with mild or moderate asthma were also excluded.

A questionnaire was used to obtain information about age, sex, duration of asthma, and the number of exacerbations in the past year, and each patient underwent physical examination.

Spirometry and bronchodilator reversibility tests were done for all patients. Skin prick tests were done for all patients with standard commercial extracts of AF (Greer, Lenoir, NC, USA). Histamine $(10 \mathrm{mg} / \mathrm{mL})$ and saline were used as positive and negative controls, respectively. Peripheral blood eosinophil count was done by standard hematoxylin and eosin staining, and an eosinophil count $>$ $450 / \mu \mathrm{L}$ was considered to indicate eosinophilia.

Total serum IgE (Monobind, Lake Forest, CA, USA), serum specific IgE (Astra Biotech, Luckenwalde, Germany), and IgG (IBL International, Hamburg, Germany) to AF were measured with an enzyme-linked immunosorbent assay according to the manufacturer's instructions. Total IgE $>200 \mathrm{IU} / \mathrm{mL}$ and specific $\mathrm{IgE}$ and specific $\mathrm{IgG}>12 \mathrm{U} / \mathrm{mL}$ were considered positive.

A recent chest X-ray and high-resolution computed tomography (HRCT) scan of the chest were analyzed to identify bronchiectasis, parenchymal fibrosis, atelectasis, and mucus plugs.

Patients with asthma who had a positive immediate reaction to the Aspergillus allergen were classified into 3 diagnostic groups: (1) AIA; (2) SAFS: patients with severe asthma, $\geq 2$ exacerbations of respiratory symptoms in the past year, and total serum IgE $<417 \mathrm{kU} / \mathrm{L}$; and (3) ABPA. The minimum diagnostic criteria for ABPA in patients with asthma included deterioration of lung function, immediate cutaneous reactivity to $\mathrm{AF}$, elevated total serum $\mathrm{IgE}>417$ $\mathrm{kU} / \mathrm{L}$ or $1000 \mathrm{ng} / \mathrm{mL}$, elevated serum IgE and/or IgG to AF, and chest X-ray infiltrate. Peripheral blood eosinophilia and central bronchiectasis on HRCT scan were considered additional diagnostic criteria for ABPA.

A total of 59 patients (39 women and 20 men, mean age $49 \pm 15$ years, age range 17-79 years) with severe asthma were included in this study. The mean duration of asthma in these patients was $11 \pm 10$ years. All patients had a history of cough, and physical examination disclosed bilateral wheezing and dyspnea at the time of the study. The number of patients with asthma who had $\geq 2$ exacerbations of symptoms in the previous year was $50(84.7 \%)$. All patients had airflow obstruction, and their $\mathrm{FEV}_{1}$ ranged from $40 \%$ to $78 \%$, and all showed reversibility in response to the bronchodilator.

The mean eosinophil count was $298 \pm 243 / \mu \mathrm{L}$ and blood eosinophilia $(450 / \mu \mathrm{L})$ was detected in 5 patients $(8.4 \%)$. Increased total serum IgE levels were observed in 20 patients (34\%) and 3 of them had a total IgE level $>417 \mathrm{kU} / \mathrm{mL}$. Serum specific IgE and IgG against AF were positive in 15 patients $(38.4 \%)$.

Infiltration was observed in chest X-rays in 15 patients $(25.4 \%)$, and permanent findings in the form of parallel lines, ring shadows and consolidation were observed in 12 patients (20\%). Chest HRCT revealed small-airway disease, bronchiectasis (3 patients with cylindrical and 1 patient with central proximal type), ground glass appearance in 4 patients, and pulmonary fibrosis in 2 patients. The prevalence of bronchiectasis in patients with asthma reportedly ranges from $18 \%$ to $52 \%(9)$.

The rate of sensitization to Aspergillus according to skin prick tests in the adult patients with severe asthma was seen in 10 patients $(17 \%)$. An earlier study from the same geographical region reported positive skin reactions to fungi in $11 \%$ ( 25 out of 230 ) of the patients with childhood asthma (10). The difference in sensitization rates may be explained by the increased environmental exposure to indoor and outdoor fungal allergens during the lifetime of older patients. González-Díaz et al. reported that the rate of sensitization to fungi was $17.1 \%$ in 480 patients with respiratory allergy according to skin tests with fungal allergens (11). Mold allergies were found in $4.3 \%$ of 115 adult Turkish patients with asthma, with Cladosporium being the most common fungus (12). A study in Saudi Arabia on 139 patients with airway allergy found positive reactions to AF in $18.2 \%$ of the patients (13), a rate similar to that in patients in the present study. The prevalence of skin test reactivity to Aspergillus in patients with asthma has been reported to vary from $16 \%$ to $45 \%$ worldwide (14-16). This variation may be explained by differences in the AF extracts used for skin tests and differences in local humidity, which is related to fungal sensitization. The natural humidity in the region under study was below than what was considered favorable to fungal growth. However, AF was found in household dust in $86 \%$ of homes in the study area, possibly because of the widespread use of air conditioners in homes (17). There was no significant relationship between age of patients and duration of asthma with a positive skin test.

The paraclinical data from these patients are summarized in Table 1. Six patients $(10.2 \%)$ with a positive skin test to Aspergillus had $\geq 2$ exacerbations of respiratory symptoms during the previous year and were diagnosed as having SAFS. Assuming that $10 \%$ of asthma cases are severe and a minimum rate of fungal sensitization of $33 \%$, about 6.5 million people would be expected to have SAFS worldwide. However, the roles of regional or genetic factors in SAFS prevalence are still unknown (18).

Only 2 patients $(3.4 \%)$ had all 4 main criteria and thus met the diagnostic criteria for of ABPA in the present study. Other studies have reported a prevalence of ABPA, ranging from $1 \%$ to $27 \%$ among patients with asthma $(6,19,20)$. For example, Al-Mobeireek et al. reported that the prevalence of ABPA was $2.7 \%$ among patients with asthma in Saudi Arabia (21). Although skin reactivity to Aspergillus in patients with asthma raises suspicions of ABPA, it is not solely confirmatory of this diagnosis. Large longitudinal 
Table 1. Diagnostic criteria of patients with positive sensitization to Aspergillus

\begin{tabular}{|c|c|c|c|c|c|c|c|c|c|c|}
\hline \multicolumn{11}{|l|}{ Patients with severe asthma Patients with severe } \\
\hline Diagnostic criteria & 1 & 2 & 3 & 4 & 5 & 6 & 7 & 8 & 9 & 10 \\
\hline Skin test reactivity to Aspergillus & + & + & + & + & + & + & + & + & + & + \\
\hline Asthma exacerbation $\geq 2$ times in past year & - & + & + & - & + & + & + & + & + & + \\
\hline Central bronchiectasis & - & - & - & - & - & - & - & + & - & - \\
\hline Chest X-ray infiltration & - & - & - & - & - & + & - & + & - & - \\
\hline Elevated specific $\operatorname{IgE}$ and $\operatorname{IgG}$ to Aspergillus & - & + & + & - & + & - & + & + & + & + \\
\hline Elevated total serum $\operatorname{IgE}$ level $>417 \mathrm{KU} / \mathrm{mL}$ & - & - & - & - & - & - & - & + & - & + \\
\hline Eosinophilia & - & - & - & - & - & + & - & + & - & + \\
\hline Diagnosis & AIA & SAFS & SAFS & AIA & SAFS & SAFS & SAFS & ABPA & SAFS & ABPA-s \\
\hline
\end{tabular}

Diagnosis

AIA SAFS SAFS AIA SAFS SAFS SAFS ABPA SAFS ABPA-S

ABPA, Allergic bronchopulmonary aspergillosis; ABPA-S, ABPA seropositive; AIS, Aspergillus-induced asthma; SAFS, Severe asthma with fungal sensitization

studies of patients with asthma and sensitization to AF may be needed to determine the progression of airway damage to better define SAFS and ABPA in the future.

According to the results, 3 patients had increased levels of total serum IgE $>417 \mathrm{kU} / \mathrm{mL}$, whereas only 2 patients were considered to have ABPA based on the standard criteria. Elevated total serum $\operatorname{IgE}>417 \mathrm{kU} / \mathrm{mL}$ is a major indicator in the diagnosis and management of ABPA. However, IgE levels may be increased in many patients with asthma but without ABPA because not all total serum IgE is directed to Aspergillus (6).

Among the patients, 2 out of 15 with high levels of Aspergillus-specific IgE were diagnosed as having ABPA and 5 were diagnosed as having SAFS. Aspergillus-specific IgE level can be considered the most sensitive test for ABPA in patients with asthma (22), but the diagnostic specificity of elevated Aspergillus-specific IgE levels in serum is limited for ABPA.

Of 10 patients with a positive Aspergillus skin test, 7 had increased levels of Aspergillus-specific IgE (kappa $=45 \%$, $\mathrm{P}<0.001)$; the agreement between these 2 tests was lower than that reported by Agarwal et al (22). One recent study emphasized that both skin test results and specific IgE measurement should be considered in the diagnosis of fungal allergy because of discordance between the test results in approximately one fourth of patients with severe asthma (23). In this connection, Cohen's kappa value showed strong concordance between positivity for serum specific IgE to Aspergillus and total $\operatorname{IgE}>200 \mathrm{IU} / \mathrm{mL}$ (kappa $=$ $73.2 \%, \mathrm{P}=0.013$ ) in the present study.

Peripheral blood eosinophilia is present in patients with ABPA who are not taking oral corticosteroids at the time of exacerbation or during the acute phase of asthma. A peripheral blood eosinophil count $>1000$ cells $/ \mu \mathrm{L}$ is often considered a diagnostic criterion, although some authors consider it to be suggestive (rather than diagnostic) of ABPA (20). None of the patients had an eosinophil count $>1000$ cells $/ \mu \mathrm{L}$. In the diagnostic criteria for ABPA recently proposed by the International Society for Human and Animal Mycology, a peripheral blood eosinophil count above 500 is considered a minor criterion which awaits prospective validation (24).

Bronchiectasis was found to be more prevalent among patients with ABPA than patients with asthma (25). One patient had central proximal bronchiectasis and another was diagnosed as having ABPA-S in this study. Results of this study were consistent with the following reports: Bronchiectasis was diagnosed in 4 patients but only 1 of them was diagnosed as having ABPA.

The prevalence of ABPA was low in this study. Considering the high prevalence of asthma and fungal sensitization in severe asthma in the area under study, physicians should be concerned about ABPA and SAFS in patients with severe asthma, especially those with a positive skin test to Aspergillus allergens.

\section{Acknowledgments}

This work was supported by a grant from Shiraz University of Medical Sciences (Grant No. 92-6337). We thank K. Shashok (Author AID in the Eastern Mediterranean) for English editing of the manuscript.

\section{Conflict of Interests}

The authors declare that they have no competing interests.

\section{References}

1. Amitani R, Kawanami R. Interaction of Aspergillus with human respiratory mucosa: a study with organ culture model. Med Mycol. 2009; 47 (Suppl 1):S127-S31.

2. Lazarus AA, Thilagar B, McKay SA. Allergic bronchopulmonary aspergillosis. Dis Mon. 2008;54(8):547-64.

3. Agarwal R, Chakrabarti A. Allergic bronchopulmonary aspergillosis in asthma: epidemiological, clinical and therapeutic issues. Future Microbiol. 2013;8(11):1463-74.

4. Denning DW, Pashley C, Hartl D, Wardlaw A, Godet C, Del Giacco $\mathrm{S}$, et al. Fungal allergy in asthma-state of the art and research needs. Clin Transl Allergy. 2014;4:14.

5. Jones AM, Horsley A, Denning DW. What is the importance of classifying Aspergillus disease in cystic fibrosis patients? Expert Rev Respir Med. 2014;8:389-92.

6. Greenberger PA, Bush RK, Demain JG, Luong A, Slavin RG, Knutsen AP. Allergic bronchopulmonary aspergillosis. J Allergy Clin Immunol Pract. 2014;2(6):703-8.

7. Agarwal R. Controversies in allergic bronchopulmonary aspergillosis. Int J Respir Care. 2010;6:53-54, 56-63.

8. National Asthma Education and Prevention Program. Expert Panel Report 3 (EPR-3): Guidelines for the Diagnosis and Management of Asthma-Summary Report 2007. J Allergy Clin Immunol. 2007; 120:S94-S138.

9. de Blic J, Scheinmann P. The use of imaging techniques for assessing severe childhood asthma. J Allergy Clin Immunol. 2007;119(4):80810 .

10. Moghtaderi M, Aleyasin S, Amin R, Kashef S. Skin test reactivity to fungal aeroallergens in asthmatic children in southern Iran. Iran J Pediatr. 2010;20(2):242-3.

11. González-Díaz SN, Arias-Cruz A, Ibarra-Chávez JA, Elizondo-Villarreal B, Rivero-Arias DM, Salinas-Díaz MR. [Prevalence of sensitization to fungi in patients with respiratory allergy]. Rev Alerg Mex. 2016;63(2):143-53.

12. Harmanci E, Metintas M, Erginel S. [Respiratory allergy to moulds among adults in Eskisehir Anatolia), Turkey]. Allerg Immunol (Paris). 2000;32(2):49-51. 
13. Almogren A. Airway allergy and skin reactivity to aeroallergens in Riyadh. Saudi Med J. 2009;30(3):392-6.

14. Fernández-Soto R, Navarrete-Rodríguez EM, Del-Rio-Navarro BE, Sienra-Monge JJL, Meneses-Sánchez NA, Saucedo-Ramírez OJ. Fungal Allergy: Pattern of sensitization over the past 11 years. Allergol Immunopathol (Madr). 2018. pii: S0301-0546(18)30044-2.

15. Maurya V, Gugnani HC, Sarma PU, Madan T, Shah A. Sensitization to Aspergillus antigens and occurrence of allergic bronchopulmonary aspergillosis in patients with asthma. Chest. 2005;127:1252-9.

16. Shah A, Panjabi C. Allergic Bronchopulmonary Aspergillosis: A Perplexing Clinical Entity. Allergy Asthma Immunol Res. 2016;8(4):28297.

17. Moghtaderi M, Farjadian S, Fereidouni M, Nasiri M, Nejat A. Indoor Dust Allergen Levels in the Homes of Patients with Childhood Asthma: An Experience from Southwestern Iran. Iran J Allergy Asthma Immunol. 2016;15(2):132-7.

18. Denning DW, Pleuvry A, Cole DC. Global burden of allergic bronchopulmonary aspergillosis with asthma and its complication chronic pulmonary aspergillosis in adults. Med Mycol. 2013,51:361-70.

19. Agarwal R, Gupta D, Aggarwal AN, Behera D, Jindal SK. Allergic bronchopulmonary aspergillosis: lessons from 126 patients attending a chest clinic in north India. Chest. 2006;130(2):442-8.

20. Shah A, Panjabi C. Allergic aspergillosis of the respiratory tract. Eur Respir Rev. 2014;23:8-29.

21. Al-Mobeireek AF, El-Rab MOGAD, Al-Hedaithy SS, Alasali K, AlMajed S, Joharjy I. Allergic bronchopulmonary mycosis in patients with asthma: period prevalence at a university hospital in Saudi Arabia. Respir Med. 2001;95(5):341-7.

22. Agarwal R, Maskey D, Aggarwal AN, Saikia B, Garg M, Gupta D et al. Diagnostic performance of various tests and criteria employed in allergic bronchopulmonary aspergillosis: a latent class analysis. PLoS One. 2013;8(4):e61105.

23. O'Driscoll BR, Powell G, Chew F, Niven RM, Miles JF, Vyas A, et al. Comparison of skin prick tests with specific serum immunoglobulin $E$ in the diagnosis of fungal sensitization in patients with severe asthma. Clin Exp Allergy. 2009;39(11):1677-83.

24. Agarwal R, Chakrabarti A, Shah A, Gupta D, Meis JF, Guleria R, et al. Allergic bronchopulmonary aspergillosis: review of literature and proposal of new diagnostic and classification criteria. Clin Exp Allergy. 2013;43:850-73.

25. Yoshida Y, Shirai T, Mikamo M, Shishido Y, Akita T, Morita S, et al. Development of allergic bronchopulmonary aspergillosis with central bronchiectasis over a 10-year period: the need to recheck allergen sensitization. Intern Med. 2013;52(18):2135-8. 